\title{
THE MASS OF WR PROGENITORS.
}

\author{
D. VANBEVEREN \\ Dept. of Physics, VUB, \\ Brussels, Belgium.
}

SUMMARY. A search through literature reveals four methods in order to derive the mass of WR progenitors, i.e.

a. WR stars must be descendant from the most massive stars which share their galactic distribution,

b. the computation of detailed evolutionary models of massive close binaries up to the WR phase, able to explain the observational constraints of these WR binaries,

c. comparing the very narrow mass-luminosity relation of massive core helium burning stars predicted by evolution and estimated bolometric luminosities of WR members of stellar aggregates,

$\mathrm{d}$. the minimum mass of the progenitor of a WR member of a cluster equals the mass of the most luminous star (or the star with the earliest spectral type) in the cluster.

Method $\mathrm{d}$ is based on a very uncertain assumption of coeval massive star formation in stellar aggregates. Even then, one may realise that method $\mathrm{d}$ is essentially similar to method a (a WR star and the most luminous stars within one cluster have similar galactic coordinates) however method a gives a statistically more significant conclusion. All methods however use different data sets and different evolutionary models and this may lead to different conclusions. I have therefore reapplied methods $\mathrm{a}, \mathrm{b}$ and $\mathrm{c}$ using the WR and OB star catalogues of van der Hucht et al. (1988, A.\&A. 199, 217), Smith and Maeder (1989, A.\&A. 211, 71), Humphreys and McElroy $(1984$, Ap.J. 284, 565) and the evolutionary models with and without convective core overshooting of Vanbeveren (1987, A.\&A. 182, 207), Maeder and Meynet $(1987, A . \& A .182,243)$, Vanbeveren (1989, A.\&A. 224, 93), Vanbeveren (1990, $A . \& A$. in press). The three methods give very similar results, i.e.

method a: on a $95 \%$ significance level the WR stars originate from massive stars with initial mass larger than $28 \mathrm{M}_{\odot}$ (non overshooting model) and $22 \mathrm{M}_{\odot}$ (with overshooting),

method b: based on detailed models for 17 well observed WR+OB binaries, it follows that WR components of close binaries originate from OB type stars with initial ZAMS mass larger than $25 \mathrm{M}_{\odot}$ (no overshooting) and $23 \mathrm{M}_{\odot}$ (with overshooting),

method c: when the bolometric correction of the majority of WR stars is larger than 4 mag (resp. $3.5 \mathrm{mag}$ ), then more than $80 \%$ of the WR stars which are member of clusters or associations originate from stars with initial ZAMS mass larger than $30 \mathrm{M}_{\odot}$ (resp. 25 $\mathrm{M}_{\odot}$ ) (no overshooting) and larger than $27 \mathrm{M}_{\odot}$ (resp. $23 \mathrm{M}_{\odot}$ ) (with overshooting). 